\title{
THE CLASSIFICATION OF SINGULAR POINTS OF ALGEBRAIC CURVES $\left({ }^{1}\right)$
}

\author{
BY
SHERWOOD EBEY
}

Introduction. To each singular point $P$ of an algebraic curve $C$ there is associated the local ring of $C$ at $P$ and the completion of this local ring, called the complete local ring at $P$. One approach to the problem of classification of singular points is to consider as equivalent all singular points whose complete local rings are isomorphic. The purpose of this paper is to obtain a scheme for the classification of the complete local rings of those singular points that are cusps or "one-branch" singularities.

This paper will be divided into five sections. In the first we show how the problem of classification of complete local rings can be translated into a problem involving the regular operation of an algebraic group on an abstract variety. The theory of algebraic groups and algebraic groups as transformation groups on a variety has been well developed in papers by Borel [1], Rosenlicht [5], and Weil [7]. Using this theory, in the second section we prove a theorem that provides the required solution. The third section records the construction of canonical forms for a nontrivial example. In the fourth section we list complete sets of canonical forms for certain simple classes of rings. In the final section we discuss the effect of the field characteristic and the problem of extending this approach to "multiple-branch" singularities.

1. The construction of the algebraic model. By a cusp we mean a singular point $P$ of the curve $C$ whose local ring $\mathfrak{D}_{P}$ is contained in one and only one valuation ring $R$ of the function field $\Omega(C)$, where $\Omega$ is the universal domain. The complete local ring $\mathfrak{D}_{P}^{*}$ at such a point is described by the following theorem.

THEOREM 1. The complete local ring of a curve $C$ at a cusp $P$ is isomorphic to a proper complete subring of the formal power series ring $\Omega[[t]]$ which contains $\Omega$ and a conductor of $\mathrm{D}_{P}^{*}$ in $\Omega[[t]]$.

Proof. If $M$ is the maximal ideal of the valuation ring $R$, it can be shown there exists an integer $n$ so that $M^{n} \subset \mathfrak{v}_{P}$. (See Theorem 2 in [6].) It follows that the topology induced on $\mathfrak{D}_{P}$ as a subset of $R$ is its own topology as a local ring. Since

Received by the editors February 4, 1964.

(1) This paper is based on the author's doctoral dissertation, written at Northwestern University under the supervision of Professor M. Rosenlicht. 
the completion of $R$ is isomorphic to $\Omega[[t]]$, the completion of $\mathfrak{D}_{P}$ may be identified with a subring of $\Omega[[t]]$. $P$ being a singular point, $\mathrm{D}_{P}^{*}$ must be a proper subring and of course contains $\Omega$.

Since $t$ may be identified with a uniformizing parameter for the valuation ring $R$ and since the quotient field of $\mathrm{o}_{P}$ is $\Omega(C)$, it follows that $t=\phi / \theta$ where $\phi$ and $\theta$ are in $\mathfrak{b}_{P}$. Considering these as power series, the orders of $\phi$ and $\theta$ are relatively prime. Thus there exists a minimal integer $d$ such that there are elements in $\mathfrak{D}_{P}$ of order $i$ for all $i \geqq d$. Since $\mathrm{o}_{P}^{*}$ is complete, it follows that $\mathrm{o}_{P}^{*}$ must contain the ideal $t^{d} \cdot \Omega[[t]]$. This ideal is the conductor of $\mathrm{o}_{P}^{*}$ in $\Omega[[t]]$, Q.E.D. The integer $d$ is called the degree of the conductor.

It should be noted that any of the rings described by Theorem 1 can be obtained from a cusp by taking polynomials in $t$ that generate the maximal ideal of the ring and using these as coordinates of a generic point for a curve.

The subring of $\Omega[[t]]$ that we have identified with the complete local ring at a cusp is not uniquely determined. We need to consider the group $\mathbb{G}$ of $\Omega$-automorphisms of the ring $\Omega[[t]]$. Each such automorphism is uniquely defined by an expression

$$
t \rightarrow u(t)=a_{0} t+a_{1} t^{2}+\cdots, \quad a_{0} \neq 0 .
$$

Thus we may identify the elements of the group $(\mathfrak{5}$ with the set of power series of order one. For any positive integer $j$, let $\mathfrak{G}_{j}$ be the normal subgroup of $\mathfrak{G}$ consisting of all automorphisms for which $u(t)$ has the form $t+a_{j} t^{j+1}+\cdots$. We recall that a connected algebraic group is solvable means it has a normal chain of connected algebraic subgroups such that each algebraic factor group is biregularly isomorphic to the additive or multiplicative group of $\Omega$. The following theorem describes the factor group $\mathfrak{G} / \mathfrak{G}_{j}$.

THEOREM 2. The group $\mathfrak{5} / \mathfrak{G}_{j}$ is a connected solvable algebraic group.

Proof. The group $\mathfrak{5} / \mathfrak{G}_{j}$ may be represented by automorphisms of the form

$$
a_{0} t+a_{1} t^{2}+\cdots+a_{j-1} t^{j}, \quad a_{0} \neq 0 .
$$

If $A^{j}$ is the affine space of dimension $j, \mathfrak{G} / \mathfrak{G}_{j}$ may be identified with the abstract variety $A^{j}-\left(a_{0}=0\right)$. The group operation that $\mathfrak{G}$ induces on the set of representatives of $\mathfrak{b} / \mathfrak{b}_{j}$ is defined rationally. Hence $\mathfrak{5} / \mathfrak{G}_{j}$ is a connected algebraic group.

The mapping $\left(a_{0} t+\cdots\right) \rightarrow\left(a_{0}\right)$ from $(5)$ onto the multiplicative group of $\Omega$ is a homomorphism with kernel $\mathfrak{G}_{1}$. The mapping $\left(t+a_{i} t^{i+1}+\cdots\right) \rightarrow\left(a_{i}\right)$ from $\mathfrak{G}_{i}$ onto the additive group of $\Omega$ is a homomorphism with kernel $\mathfrak{G}_{i+1}$. It follows that the group $\left(\mathfrak{b} / \mathfrak{G}_{j}\right.$ is a solvable algebraic group. Q.E.D.

The degree $d$ of the conductor is clearly an invariant for the group $\mathfrak{b}$ operating on the set of rings described by Theorem 1. If we consider the subclass of those 
rings with a fixed $d$, then each automorphism of $\mathfrak{b}_{d}$ operates as the identity on this subclass. Thus we may replace the group $(5)$ with the solvable connected algebraic group $\mathfrak{5} / \mathfrak{G}_{d}$.

It should be noted we are here using the term " $\mathfrak{G}$ operates on a set of rings" in the sense of abstract group theory. There remain two steps to complete our algebraic model. The first is to prove that a certain subclass of rings (determined by some numerical invariants) may be identified with an abstract variety. Then we will show that the operation of the factor group $\mathfrak{G} / \mathfrak{G}_{d}$ is in the sense of algebraic geometry.

In what follows we will find it convenient to use the concept of constructible set, introduced by Chevalley in [3]. A subset $H$ of an abstract variety $V$ is constructible means it is the finite union of sets, each of which is the intersection of two sets, one an open set and one a closed set in the Zariski topology for $V$. Consstructible sets have the following properties: The union and intersection of two constructible sets are constructible, the complement in $V$ of a constructible set is constructible, and if $H$ is a constructible subset of $E \times F$, the projection of $H$ on $E$ is a constructible subset of $E$.

For each subring $\mathfrak{D}$ of $\Omega[[t]]$ there corresponds a semigroup of integers $G(\mathfrak{D})$ consisting of the orders of elements of $\mathfrak{b}$. Each semigroup for the rings we are considering is a proper subset of the positive integers and contains a minimal integer $d$ such that $i \geqq d$ implies $i$ is in $G$. By analogy we will call $d$ the conductor of the semigroup. It is clear that $G(\mathfrak{D})$ is an invariant under the group of automorphisms 65 .

Let $G$ be a fixed semigroup of positive integers with a conductor $d$. Let $\mathfrak{I}$ be the class of all complete local subrings of $\Omega[[t]]$ such that $G(\mathfrak{D})=G$. Our next step is to show that the class of rings $\mathfrak{I}$ may be represented by a constructible subset of an affine space.

Let $n_{1}<n_{2}<\cdots<n_{r}$ be the sequence of integers in $G$ that are less than $d$. Then for each $i$ let $m_{i 1}<m_{i 2}<\cdots<m_{i s_{i}}$ be the sequence of integers between $n_{i}$ and $d$ that are not in $G$. The following lemma is immediate.

Lemma 1. For every ring $\mathfrak{D}$ in $\mathfrak{I}$ and for each $i, 1 \leqq i \leqq r$, there exists an element $\phi_{i}(t)$ in $\mathfrak{D}$ of the form

$$
\phi_{i}(t)=t^{n_{i}}+a_{i 1} t^{m_{i 1}}+a_{i 2} t^{m_{i 2}}+\cdots+a_{i s_{i}} t^{m_{i s_{i}}}
$$

and the set of constants

$$
(a)=\left(a_{11}, \cdots, a_{1 s_{1}}, \cdots, a_{r s_{r}}\right)
$$

is uniquely determined by the ring $\mathfrak{o}$.

Lemma 1 implies we have an injection of the class of rings $\mathfrak{I}$ into the affine space $A^{j}\left(j=\Sigma s_{i}\right)$. Let $H$ be the image of $\mathfrak{I}$ under this map. We will now describe $H$. 
THEOREM 3. The set $H$ is a constructible subset of $A^{j}$.

Proof. First let $(a)$ be a generic point of $A^{j}$ over the prime field $K$. Let $(\phi(a, t))$ be the set of $r$ polynomials of form (1). Then for each $(\xi)$ in $A^{j}$ we define the ring $\mathfrak{D}(\xi)$ as the complete local subring of $\Omega[[t]]$ generated by the set of polynomials $(\phi(\xi, t))$ and the ideal $t^{d} \cdot \Omega[[t]]$. If $\mathfrak{D}$ is any ring in $\mathfrak{I}$ and $(\xi)$ is the point of $H$ corresponding to $\mathfrak{D}$ by the mapping defined by Lemma 1 , then $\mathfrak{D}(\xi)=\mathfrak{v}$.

Let $G(\xi)$ be the semigroup of positive integers corresponding to the ring $\mathfrak{p}(\xi)$. For each $(\xi)$ in $A^{j}, G \subset G(\xi)$. The elements of $H$ can be characterized in this way: $(\xi)$ is in $H$ if and only if $G(\xi)=G$.

If $v$ is any integer not in $G$ and greater than $n_{1}$, define $F_{v}$ as the subset of $A^{j}$ such that $(\xi)$ belongs to $F_{v}$ if and only if $v$ belongs to $G(\xi)$. If each $F_{v}$ were constructible, then $H$ would be constructible because $H$ can be described as the intersection of the complement in $A^{j}$ of the sets $F_{v}$. Thus we are reduced to proving $F_{v}$ is a constructible set.

Consider the set of expressions $\{\theta(x, a, t)\}$ where each is of the form

$$
\theta(x, a, t)=\sum_{k=1}^{l} x_{k} M_{k}
$$

where the $x$ 's are variables and each $M_{k}$ is some power product of polynomials in the set $(\phi(a, t))$. Also we assume each $M_{k}$ in (2) is of order $\leqq v$ with respect to $t$. Thus the set of such $\theta$ is finite.

For each $\theta$ define the set $F_{v \theta}$ as the set of all $(\xi)$ in $A^{j}$ for which there exist constants $(c)=\left(c_{1}, \cdots, c_{l}\right)$ so that $\theta(c, \xi, t)$ has order $v$. Since $F_{v}$ is the union of the sets $F_{v \theta}$, we are reduced to proving $F_{v \theta}$ is a constructible set.

In $\theta(x, a, t)$ let $L_{1}(a, x), \cdots, L_{s}(a, x)$ be the coefficients of powers of $t$ of degree $<v$ and let $L_{s+1}(a, x)$ be the coefficient of $t^{\nu} . F_{v \theta}$ is the set of all $(\xi)$ for which the linear form $L_{s+1}(\xi, x)$ is linearly independent of the forms $L_{1}(\xi, x), \cdots, L_{s}(\xi, x)$.

Let $B(a)$ be the matrix of coefficients of the forms $L_{1}, \cdots, L_{s}$ and $D(a)$ the matrix for the forms $L_{1}, \cdots, L_{s+1}$. Denote by $B^{(i)}(a)$ and $D^{(i)}(a)$ the determinants of the $i \times i$ minors of these matrices. $(\xi)$ is in $F_{v \theta}$ if and only if the rank of $D(\xi)$ is one more than the rank of $B(\xi)$. Thus $F_{v \theta}$ is the finite union of sets of the form

$$
F_{m}=\left[\bigcap_{i>m}\left(B^{(i)}(a)=0\right)\right]-\left[\bigcap_{i=m}\left(B^{(i)}(a)=0\right)\right]-\left[\bigcap_{i=m+1}\left(D^{(i)}(a)=0\right)\right] .
$$

Since each $F_{m}$ is a constructible set, this completes the proof of Theorem 3.

We recall that a connected algebraic group $D$ operates regularly on an abstract variety $V$ means that there is an operation in the sense of group theory and this operation is given by an everywhere defined rational map from $D \times V$ onto $V$. The following theorem will complete our algebraic model.

THEOREM 4. There exists a regular operation of $\mathfrak{b} / \mathfrak{G}_{d}$ on $A^{j}$ which is invariant 
on $H$ and such that on $H$ the operation is the same as that induced from the operation of $\mathfrak{5} / \mathfrak{G}_{d}$ on the class of rings $\mathfrak{I}$.

Proof. As before let $(a)$ be a generic point of $A^{j}$ over the prime field $K$ and for each $i, 1 \leqq i \leqq r$, let $\phi_{i}(a, t)$ be the polynomial defined by expression (1). Let $\alpha(t)=\alpha_{0} t+\alpha_{1} t^{2}+\cdots, \alpha_{0} \neq 0$, represent an element of $\mathfrak{b} / \mathfrak{G}_{d}$ which is generic over $K(a)$.

To define the operation of $\mathfrak{5} / \mathfrak{G}_{d}$ on $A^{j}$ we define $\alpha(a)$ by the polynomials

$$
\phi_{i}(\alpha(a), t)=\frac{1}{\alpha_{0}^{n_{i}}} \phi_{i}(a, \alpha(t))-\sum_{k>i} P_{i k}(a, \alpha) \phi_{k}(a, \alpha(t))-\theta
$$

where (A) $\theta$ is an element of $\Omega[[t]]$ of order $\geqq d$ chosen so that the right side of (3) has no powers of $t$ of degree $\geqq d$, (B) $P_{i k}(a, \alpha)$ is the quotient of a polynomial in $K[a, \alpha]$ by a power of $\alpha_{0}$, and (C) the expressions $P_{t k}(a, \alpha)$ are uniquely determined by the requirement that the right side of (3) have a zero coefficient for each power $t^{n_{i}}, k>i$.

The operation $(\alpha, a) \rightarrow \alpha(a)$ is an everywhere defined rational map of $\mathbb{G} / \mathfrak{G}_{d} \times A^{j}$ onto $A^{j}$. If $\beta$ is a second element of $\left(\mathfrak{G} / \mathfrak{G}_{d}\right.$ and $\beta \alpha$ is the automorphism which is the product of $\alpha$ followed by $\beta$, by direct computation it follows that $\beta \alpha(a)=\beta(\alpha(a))$. Thus (3) defines a regular operation of $\left(5 / \mathfrak{G}_{d}\right.$ on $A^{j}$.

Now consider $(\xi)$ in $H . \mathfrak{D}(\xi)$ is generated by the set of polynomials $\{\phi(\xi, t)\}$ plus the ideal $C=t^{d} \cdot \Omega[[t]] \cdot \mathfrak{D}(\alpha(\xi))$ is the complete local ring generated by the polynomials $\{\phi(\alpha(\xi), t)\}$ and $C$. But (3) implies that $\{\phi(\xi, \alpha(t))\}$ plus $C$ is also a set of generators for the ring $\mathfrak{D}(\alpha(\xi))$. Therefore $\mathfrak{p}(\alpha(\xi))$ is in the class $\mathfrak{I}, \alpha(\xi)$ is in $H$, and the set $H$ is invariant under this operation. Q.E.D.

We have now constructed a model in which we have a solvable connected algebraic group operating regularly on an abstract variety. This is a situation that belongs to the theory of algebraic groups and is described by the following theorem.

THEOREM 5. If $\mathfrak{H}$ is a connected solvable algebraic group operating regularly on an abstract variety $V$, there exists a constructible subset $W$ of $V$ such that for each $v$ in $V$ there exists a unique $w$ in $W$ such that $v$ is in $\mathfrak{S} w$.

This theorem will be proved later, after we have completed the discussion of the complete local subrings of $\Omega[[t]]$.

By applying Theorem 5 to the operation of $\mathfrak{F} / \mathfrak{G}_{d}$ on $A^{j}$ and by considering $W_{1}=W \cap H$, we derive the following result.

THEOREM 6. There exists a constructible subset $W_{1} \subset A^{j}$ such that for any ring $\mathfrak{D}$ in $\mathfrak{I}$ there exists a unique element $(\xi)$ in $W_{1}$ so that $\mathfrak{D}$ and the ring $\mathfrak{D}(\xi)$ are equivalent by the group $\mathbf{6}$.

If $\mathfrak{v}$ is a complete local ring contained in $\Omega[[t]]$, then $\theta_{1}, \cdots, \theta_{s}$ is a minimal set of generators which means that the ring generated by a proper subset will be a 
proper subring. This is equivalent to the assertion that $\theta_{1}, \cdots, \theta_{s}$ represents a basis for the $\Omega$-vector space $M / M^{2}, M$ being the maximal ideal of $\mathfrak{D}$. The number of elements in a minimal set of generators for $\mathfrak{v}$ is an invariant under the operation of the group.

Theorem 6 implies that each equivalence class of rings in $\mathfrak{I}$ is uniquely represented by the coefficients of $r$ polynomials in a ring $\mathfrak{D}(\xi)$ where $(\xi)$ is in the constructible subset $W_{1}$. We intend to improve the statement of Theorem 6 , by reducing the set of polynomials representing $\mathfrak{D}(\xi)$ to a minimal set of generators of $\mathfrak{D}(\xi)$ and so that the coefficients of these polynomials will be parametrized by a constructible set in an affine subspace of $A^{j}$.

To each ring $\mathfrak{D}$ in $\mathfrak{I}$ there is associated an increasing sequence of integers $m(\mathfrak{D})=\left(m_{1}, \cdots, m_{s}, \cdots, m_{l}\right)$ where this is the set of all integers in the semigroup $G$ such that $\mathfrak{D}$ has no elements in $M^{2}$ of order $m_{i}$. We denote by $m_{s}$ the integer of $m(\mathfrak{D})$ such that $m_{s}<d$ and $m_{s+1} \geqq d$. $m(\mathfrak{D})$ is an invariant under the operation of $\mathfrak{G}$ and there are only a finite number of sets possible for the rings in $\mathfrak{T}$. Thus $\mathfrak{I}$ is partitioned into a finite number of invariant subclasses by the sets of integers $m(\mathfrak{D})$.

Let $(m)=\left(m_{1}, \cdots, m_{s}, \cdots, m_{l}\right)$ be one of the sets described in the previous paragraph. Define $\mathfrak{I}(m)$ as the subclass of rings $\mathfrak{D}$ in $\mathfrak{I}$ so that $m(\mathfrak{D})=(m)$. The following lemma proceeds directly from the definitions.

LEMMA 2. If $\mathfrak{D}$ is any ring of $\mathfrak{I}(m)$ and if $\left(\theta_{1}, \cdots, \theta_{l}\right)$ is any set of elements of $\mathfrak{D}$ such that each $\theta_{i}$ is of order $m_{i}$, respectively, then $\left(\theta_{1}, \cdots, \theta_{l}\right)$ is a minimal set of generators for $\mathfrak{D}$.

The set of integers $(m)$ must be a subset of the set of integers $(n)$ which form a minimal set of generators for the semigroup $G$. Suppose $v$ is in $(n)$ and $v<d$. Then $v$ is one of the integers $n_{i}$ and there will be an element of order $v=n_{i}$ in $M^{2}$ of $\mathfrak{D}(\xi)$ if and only if $n_{i}$ is in the semigroup of integers corresponding to the complete local ring generated by $\left(\phi_{1}(\xi, t), \cdots, \phi_{i-1}(\xi, t)\right)$. If $v \geqq d$ there will be an element of order $v$ in $M^{2}$ of $\mathfrak{D}(\xi)$ if and only if $v$ is in the semigroup for the ring generated by $\left(\phi_{1}(\xi, t), \cdots, \phi_{r}(\xi, t), t^{d}, \cdots, t^{\nu-1}\right)$.

The scheme used to prove Theorem 3 is applicable here and implies that for each such $v$ there exists a constructible subset $F_{v}$ of $A^{j}$ such that there is an element of order $v$ in $M^{2}$ of $\mathfrak{D}(\xi)$ if and only if $(\xi)$ is in $F_{v}$. Thus $\mathfrak{D}(\xi)$ belongs to $\mathfrak{I}(m)$ if and only if $(\xi)$ is not in $F_{v}$ for $v$ in $(m)$ and $(\xi)$ is in $F_{v}$ for the $v$ 's not in $(m)$. Hence we have proved the following lemma.

LEMMA 3. The subset $H_{1}$ of $H$ which consists of all $(\xi)$ in $A^{j}$ such that $\mathfrak{D}(\xi)$ is in $\mathfrak{I}(m)$ is a constructible subset of $A^{j}$.

Now if we define $W_{2}=H_{1} \cap W_{1}$ where $W_{1}$ is the set described in Theorem 6, then for each $\mathfrak{D}$ in $\mathfrak{T}(m)$ there exists a unique $(\xi)$ in $W_{2}$ such that $\mathfrak{D}$ is equivalent to $\mathfrak{D}(\xi)$ by the group $\left(\mathfrak{b}\right.$. Denote by $A^{k}$ the affine subspace of $A^{j}$ which represents 
the coefficients of those polynomials $\phi_{i}(a, t)=t^{n_{i}}+\cdots$ for which $n_{i}$ appears in the set $(m) . W_{2}$ being constructible, if $Z$ is the projection of $W_{2}$ on $A^{k}, Z$ is a constructible subset of $A^{k}$. Furthermore the projection on $W_{2}$ on $A^{k}$ is one-to-one. This completes the proof of the improved version of Theorem 6. Before we summarize these results as Theorem 7, we will restate the symbols and definitions needed to express this theorem.

$\mathfrak{I}(m)$ is the class of all complete local subrings $\mathfrak{D}$ of $\Omega[[t]]$ with a conductor and satisfying: (1) For each $\mathfrak{D}$, the semigroup $G(\mathfrak{D})$ is a fixed semigroup $G$ with conductor $d>0$. (2) $(m)=\left(m_{1}, \cdots, m_{l}\right)$ are the integers of $G$ that are the orders of a minimal set of generators of $\mathfrak{D}$. $\left(m_{s}<d\right.$ and $m_{s+1} \geqq d$.)

Then for each $m_{i}, 1 \leqq i \leqq s$, let $p_{i 1}, \cdots, p_{i r_{i}}$ be the sequence of integers between $m_{i}$ and $d$ that are not in $G$ and define the polynomials.

$$
\theta_{i}(b, t)=t^{m_{i}}+b_{i 1} t^{p_{i 1}}+\cdots+b_{i r_{i}} t^{p_{i r_{i}}}
$$

Then $A^{k}\left(k=\sum r_{i}\right)$ is the affine space of coefficients for the set of polynomials $(\theta(b, t))$. The final result of this discussion is the following.

THEOREM 7. There exists a constructible subset $Z$ of $A^{k}$ such that for any ring $\mathfrak{D}$ in $\mathfrak{I}(m)$ there is a unique point $\left(b^{\prime}\right)$ in $Z$ so that $\mathfrak{D}$ is equivalent by the group $(5)$ of $\Omega$-automorphisms of $\Omega[[t]]$ to the complete local ring with a minimal set of generators

$$
\left(\theta_{1}\left(b^{\prime}, t\right), \cdots, \theta_{s}\left(b^{\prime}, t\right), t^{m_{s+1}}, \cdots, t^{m l}\right) .
$$

2. A theorem concerning algebraic groups. In this section we will prove Theorem 5. This theorem belongs to the theory of algebraic groups which has been definitively outlined in papers by Borel, Rosenlicht, and Weil. In the proof we will use freely the terminology and results of [5] and [7]. We recall that $\mathfrak{H}$ is a connected solvable algebraic group operating regularly on an abstract variety $V$ and we are to find a constructible subset $W$ of $V$ so that for each $v$ in $V$ there is a unique $w$ in $W$ with $v$ in $\mathfrak{H} w$,

Induction on $\operatorname{dim}(V)$ provides a proof of the following preliminary remark: If $F$ is a proper, closed subset of $V$ such that $\mathfrak{H} F=F$, then it will suffice to prove the theorem for the abstract variety $V-F$,

Then let $T$ be the variety of $\mathfrak{G}$-orbits on $V$ and $\tau$ the natural rational map from $V$ to $T$ (cf. Theorem 2 of [5]). $T$ is uniquely determined up to a birational correspondence. By a proposition of Weil's (cf. Appendix, Proposition 8 of [7]) there is a closed subset $F$ of $V$ such that $\tau$ is a regular on $V-F$. We will show $F$ may be chosen invariant under $\mathfrak{H}$.

Let $g$ be in $\mathfrak{H}$ and $k$ a field of definition for $\mathfrak{H}, V$, and the operation of $\mathfrak{G}$ on $V$. Let $v$ be a generic point for $V$ over $k(g)$. Since $k(g, v)=k(g, g v), g v$ is generic for $V$ over $k(g)$. Therefore by the fundamental property defining $\tau$ and $T, \tau(v)=\tau(g v)$. Thus the rational map $\tau$ equals the rational map $\tau g$ on $V$. Let $\tau$ be regular at a point 
$p$. Then $\tau g$ is regular at $p$ and $\tau$ is regular at $g p$. It also follows that $\tau(p)=\tau(g p)$. Thus $\tau$ is regular at $p$ if and only if $\tau$ is regular at $g p$.

Since $\tau$ is regular on $V-F$, then $\tau$ must be regular on $V-g F$ for any $g$ in $\mathfrak{H}$. If we let $F_{1}=\bigcup g F$ it follows that $\tau$ is regular on $V-F_{1}$ where $F_{1}$ is a proper closed subset of $V$ that is invariant under $\mathfrak{H}$. Then applying our preliminary remark, it suffices to prove the theorem for an abstract variety $V$ which satisfies the additional assumption that the rational map $\tau$ from $V$ to the variety of $\mathfrak{H}$ orbits $T$ is regular on $V$.

The hypothesis that $\mathfrak{H}$ is a solvable connected algebraic group operating regularly on $V$ implies the existence of a cross-section $\sigma$, i.e. $\sigma$ is a rational map, $\sigma: T \rightarrow V$ such that $\tau \sigma=1$ (cf. Theorem 10 in [5]).

There exists a smallest, proper closed subset $T_{1} \subset T$ such that $\sigma$ is regular on $T-T_{1}$. Let $V^{\prime}=V-\tau^{-1}\left(T_{1}\right)$ and $T^{\prime}=T-T_{1} \cdot \tau^{-1}\left(T_{1}\right)$ is a proper closed subset of $V$ that is invariant under $\mathfrak{H}, \sigma\left(T^{\prime}\right) \subset V^{\prime}, T^{\prime}$ is a variety of $\mathfrak{H}$-orbits on $V^{\prime}$, $\tau$ is regular on $V^{\prime}$ and the cross-section $\sigma$ is regular on $T^{\prime}$. Thus we are reduced to proving the theorem under the additional assumptions that $\tau$ is regular on $V$ and $\sigma$ is regular on $T$.

The cross-section $\sigma$ is a rational map from $T$ to the variety $\overline{\sigma(T)} . \sigma$ is regular on $T$ and generically surjective on $\overline{\sigma(T)}$. Another proposition of Weil's (cf. Appendix, Proposition 10 of [7]) asserts that under these conditions there exists a set $W \subset \sigma(T) \subset \overline{\sigma(T)}$ such that $W$ is an open dense subset of $\overline{\sigma(T)}$,

Let $k$ be a field of definition for $\mathfrak{H}, V$, solvability of $\mathfrak{H}$, operation of $\mathfrak{H}$ on $V, \tau, \sigma$, $\overline{\sigma(T)}$, and for the components of the complement of $W$ in $\overline{\sigma(T)}$.

Now $W$ may be considered as an abstract variety, defined over $k$. Thus $g \times w \rightarrow g w$ is a rational map from $\mathfrak{S} \times W \rightarrow V$ that is regular on $\mathfrak{H} \times W$ and defined over $k$. There is a point $p$ which is generic for $\overline{\sigma(T)}$ over $k$ (and hence $p$ is in $W$ ) and an element $g$ in $\mathfrak{H}$ such that $g p$ is generic for $V$ over $k$ (cf. Theorem 10 in [5]). It follows that the rational map $\mathfrak{H} \times W \rightarrow V$ is generically surjective. Thus there exists a proper closed subset $F$ of $V$ such that $V^{\prime}=V-F \subset \mathfrak{H} W$. By replacing $F$ by $\bigcup g F$ if necessary we may suppose that $F$ is invariant under $\mathfrak{H}$. Then it suffices to prove the theorem for this abstract variety. (This is the third and final reduction.)

If we define $W^{\prime}$ as the intersection of $W$ with the complement of $F$ in $V$ then $V^{\prime}=\mathfrak{S} W^{\prime}$ follows by straightforward computation. We assert that $W^{\prime}$ is the required constructible subset of $V^{\prime}$. Suppose $p$ is in $V^{\prime}$ with $p=g_{1} w_{1}^{\prime}=g_{2} w_{2}^{\prime}$ where the $w_{i}^{\prime}$ are in $W^{\prime}$. Now $W^{\prime} \subset W \subset \sigma(T)$ implies $w_{i}^{\prime}=\sigma\left(t_{i}\right)$ with $t_{i}$ in $T$. Then for $i=1,2, \tau(p)=\tau\left(g_{i} w_{i}^{\prime}\right)=\tau\left(w_{i}^{\prime}\right)=\tau\left(\sigma\left(t_{i}\right)\right)=t_{i}$. Thus $t_{1}=t_{2}$ and $w_{1}^{\prime}=w_{2}^{\prime}$. We have shown that each element of $V^{\prime}$ is equivalent by the group $\mathfrak{H}$ to a unique element of $W^{\prime}$. This completes the proof of Theorem 5 of the first section.

3. The construction of canonical forms for an example. We have proved the existence of a solution to the problem of finding canonical forms for the generators 
of complete local subrings $\mathfrak{D}$ of $\Omega[[t]]$ that have a conductor. The answer is that the coefficients occuring in these canonical forms will be parametrized by a union of constructible subsets of the corresponding affine space.

The general theory gives no indication of methods to use in the construction of these constructible subsets of the affine space of coefficients. Moreover a single point as well as affine varieties and affine subspaces are in the category of constructible subset. The general theory gives no hint concerning the possibility of the constructible subsets being of a special type.

In this section we will compute these constructible subsets for a specific example. We are including these computations for three purposes: first, to illustrate elementary devices that can be used to compute canonical forms; second, to indicate that these computed canonical forms do not yield affine subspaces or affine varieties in every instance; third, to compare the difference in effect of automorphisms of the form $t \rightarrow c t, c \neq 0$, and those of the form $t \rightarrow t+c t^{\nu+1}+\cdots$.

Let $\mathfrak{I}$ be the class of complete local subrings $\mathfrak{D}$ of $\Omega[[t]]$ such that $\mathfrak{D}$ has a minimal set of generators consisting of a pair $(u(t), v(t))$ with order $(u)=5$ and $\operatorname{order}(v)=9$. By using a step-by-step procedure we will calculate canonical forms for this class of rings.

The semigroup $G$ consisting of the orders of elements of $\mathfrak{D}$ is

$$
G=\{5,9,10,14,15,18,19,20,23,24,25,27,28,29,30,32, n>32\} .
$$

Now certain integers will arise in the computation and the forms we compute will hold for all characteristics $p$ prime to these integers. However to avoid enumeration of special cases, we will simply assume characteristic $=0$.

Since $\Omega[[t]]$ is complete, we can find a power series $u_{1}(t)$ so that $u_{1}^{5}=u$. Applying the automorphism $t \rightarrow u_{1}(t)$ we have that each ring in $\mathfrak{I}$ is equivalent to a ring with first generator $t^{5}$. Moreover let $v$ be in $G, v=i 5+j 9$ where $i, j$ are non-negative integers. If $v(t)$ has the term $c t^{v}, c \neq 0$, then the pair

$$
\left(u(t), v(t)-c u^{i}(t) v^{j}(t)\right)
$$

is also a set of generators for $\mathfrak{D}$. Hence we have proved that any ring in $\mathfrak{I}$ is equivalent to a ring with a pair of generators of the form $A$ :

$$
\left(t^{5}, t^{9}+a t^{11}+b t^{12}+c t^{13}+d t^{16}+e t^{17}+f t^{21}+g t^{22}+h t^{26}+i t^{31}\right) .
$$

We will note that each pair of generators of form $A$ determines a distinct ring. The problem is to find a subset of rings of this form so that each ring with generators of form $A$ is equivalent to one and only one ring in the subset. We will first consider the group $\mathfrak{G}_{1}$ of automorphisms of the form $t \rightarrow t+\cdots$. An automorphism of the form $t \rightarrow t+b_{v} t^{v+1}+\cdots, b_{v} \neq 0$, will be said to be an unipotent automorphism of height $v$.

Before we begin the computation of canonical forms for rings with generators 
of form $A$ we will prove three lemmas. Each of these describes a device used to reduce form $A$ to canonical form. It will be convenient to describe each of these only once. Let $m_{1}<m_{2}<\cdots<m_{s}$ be the sequence of integers between 9 and 32 that are not in $G$.

LeMma 1. If $5+m_{j}-9$ is in $G$, then any ring $\mathfrak{D}_{1}$ with generators of the form

$$
\left(t^{5}, t^{9}+a_{1} t^{m_{1}}+\cdots+a_{j-l} t^{m_{j-1}}+a_{j} t^{m_{j}}+\cdots\right)
$$

is equivalent to a ring with generators of the same form with $a_{j}=0$ and $a_{k}$ unchanged for $k<j$.

Proof. Apply the automorphism $t \rightarrow t-(1 / 9) a_{j} t^{\left(m_{j}-9\right)+1}$. The image of the ring $\mathfrak{D}_{1}$ is a ring $\mathfrak{D}_{2}$ with generators

$$
\left(t^{5}-\frac{5}{9} a_{j} t^{5+m_{j}-9}+\cdots, t^{9}+a_{1} t^{m_{1}}+\cdots+a_{j-l} t^{m_{j-1}}+0 \cdot t^{m_{j}}+\cdots\right) .
$$

Since $5+m_{j}-9$ is in $G$, this power of $t$ can be removed from the first generator. Then by a second unipotent automorpism (of height $v>m_{j}-9$ ) we obtain a ring $\mathrm{o}_{3}$ with generators of form $A$. For $\mathfrak{D}_{3},\left(a_{1}, \cdots, a_{j-1}\right)$ will be the same as for $\mathrm{o}_{1}$ and $a_{j}$ will be 0 .

LEMMA 2. If $m_{k}=m_{j}+i \cdot 5$, then any ring $\mathfrak{D}_{1}$ with generators of the form

$$
\left(t^{5}, t^{9}+a_{j} t^{m_{j}}+\cdots\right) \quad \text { with } a_{j} \neq 0
$$

is equivalent to a ring with generators of the same form with $a_{k}=0$ and $a_{l}$ unchanged for $l<k$.

Proof. Apply the automorphism $t \rightarrow t+Z t^{1+i \cdot 5}$. The image of the ring $\mathfrak{D}_{1}$ is a ring $\mathfrak{D}_{2}$ with generators of the form

$$
\left(t^{5}, t^{9}+a_{j} t^{m_{j}}+9 Z t^{9+5 i}+\left[a_{j} m_{j} Z+a_{k}\right] t^{m_{k}}+\cdots\right) .
$$

Subtracting $9 Z \cdot t^{i 5} \cdot\left[t^{9}+\cdots\right]$ from the second generator gives a second generator of the form

$$
t^{9}+a_{j} t^{m_{j}}+\cdots+\left[a_{j}\left(m_{j}-9\right) Z+a_{k}\right] t^{m_{k}}+\cdots .
$$

Since $\left(m_{j}-9\right) a_{j} \neq 0$, we can choose $Z$ so the coefficient of $t^{m_{k}}$ is 0 while the $a_{l}$ remain unchanged for $l<k$.

LEMMA 3. Any ring $\mathfrak{D}_{1}$ with generators of the form

$$
\left(t^{5}, t^{9}+b t^{12}+d t^{16}+f t^{21}+\cdots\right) \quad \text { with } b \neq 0
$$

is equivalent to a ring with generators of the same form with $f=0$ and $b, d$ unchanged. 
Proof. Apply the automorphism $t \rightarrow t+Z t^{9+1}$. The image of $\mathfrak{D}_{1}$ is a ring $\mathfrak{D}_{2}$ with generators of the form

$$
\left(t^{5}+, 5 Z t^{14}+\cdots, t^{9}+b t^{12}+d t^{16}+9 Z t^{18}+[12 b Z+f] t^{21}+\cdots\right)
$$

The element of order 14 in this ring is $t^{14}+b t^{17}+\cdots$ and the element of order 18 is $t^{18}+2 b t^{21}+\cdots$. Hence $\mathfrak{b}^{2}$ will have generators of that form

$$
\left(t^{5}-5 b Z t^{17}+\cdots, t^{9}+b t^{12}+d t^{16}+[-6 b Z+f] t^{21}+\cdots\right) .
$$

Now apply to $\mathfrak{D}_{2}$ the automorphism $t \rightarrow t+Y t^{12+1}$. Then the ring $\mathfrak{D}_{3}$ will have generators of the form

$$
\left(t^{5}+[5 Y-5 b Z] t^{17}+\cdots, t^{9}+b t^{12}+d t^{16}+[9 Y-6 Z+f] t^{21}+\cdots\right) .
$$

We can choose $Y$ and $Z$ so the coefficient of $t^{17}$ in the first generator and the coefficient of $t^{21}$ in the second generator are both 0 . Then by applying a final unipotent automorphism of height $v>12$, we can obtain a ring $\mathfrak{D}_{4}$ with generators in the original form with $f=0$ and with $b, d$ unchanged. This completes the proofs of these three lemmas.

If the image of a ring $\mathfrak{D}$ with generators of form $A$ by a unipotent automorphism $t \rightarrow t+c_{v} t^{v+1}+\cdots$ is a ring of the same form, then $v>3$. (For otherwise the first generator could not be $t^{5}$.)

By Lemma 1 we can obtain an equivalent ring with generators of form $B$ :

$$
\left(t^{5}, t^{9}+a t^{11}+b t^{12}+d t^{16}+e t^{17}+f t^{21}+g t^{22}+h t^{26}+i t^{31}\right) .
$$

If a unipotent automorphism transforms a ring of this form into a ring of the same form, then $v>4$. (For if $v=4$, the second generator would have a term $t^{13}$.) Now $a$ and $b$ are invariants for the operation of $\mathfrak{b}_{1}$ on these rings and it is necessary now to divide the consideration into three cases - Case 1: $a \neq 0$, Case 2: $a=0$, $b \neq 0$, Case 3: $a=0, b=0$.

Case 1: $a \neq 0$. By applying Lemma 2, we obtain an equivalent ring of the same form with $d=0$. Then for a ring of this form $(d=0)$ to remain in the same form, $v>5$. Now $v \neq 6,7$, or 8 for otherwise $t^{5}$ could not be the first generator. Hence the only unipotent automorphisms leaving form $B$ unchanged where $a \neq 0$ and $d=0$ are those with $v>8$. This means the constants $a, b, e$ are invariants for unipotent automorphisms.

Then to conclude Case 1 , by alternating the applications of Lemmas 2 and 1 , we can obtain an equivalent ring with generators of form $B$ and with $f=g=h=i=0$. Hence any ring of form $B$ with $a \neq 0$ is equivalent to a ring with generators of the form

$$
\left(t^{5}, t^{9}+a t^{11}+b t^{12}+e t^{17}\right)
$$

and none of these rings can be equivalent by unipotent automorphisms. 
Case 2: $a=0, b \neq 0$. By applying Lemma 2, we obtain an equivalent ring of form $B$ with $e=0$. Then for a ring of this form $(a=0, e=0)$ to remain in the same form by any unipotent automorphism, $v>8$. This implies the constants $b$ and $d$ are invariants for unipotent automorphisms.

By applying Lemma 3, we obtain an equivalent ring of the same form with $f=0$. By applying Lemma $1, g=0$. By the same computational scheme used in Lemma 3, $h=0$. (This time the initial automorphism would be $t \rightarrow t+Z t^{14+1}$.) Finally by Lemma $1, i=0$. Hence we have shown that any $\operatorname{ring}$ of form $B$ with $a=0$, and $b \neq 0$ is equivalent to a ring with generators of the form

$$
\left(t^{5}, t^{9}+b t^{12}+d t^{16}\right)
$$

and moreover none of these rings is equivalent by unipotent automorphisms.

Case 3: $a=0, b=0$. We are now considering the set of rings with generators of form $C$ :

$$
\left(t^{5}, t^{9}+d t^{16}+e t^{17}+f t^{21}+g t^{22}+h t^{26}+i t^{31}\right) .
$$

From our arguments above, we know that the only unipotent automorphism of height $v<9$ that would leave form $C$ invariant would be $t \rightarrow t+Z t^{5+1}$. But such an automorphism would leave $d$ and $e$ invariant. Thus we may divide Case 3 into three subcases: Case 3.1: $d \neq 0$, Case 3.2: $d=0, e \neq 0$, Case 3.3: $d=e=0$.

Case 3.1: form $C$ with $d \neq 0$. By alternate applications of Lemmas 2 and 1, any ring with generators of form $C$ and $d \neq 0$ is equivalent to a ring with generators of the form

$$
\left(t^{5}, t^{9}+d t^{16}+e t^{17}\right)
$$

and moreover none of these can be equivalent by unipotent automorphisms.

Case 3.2: form $C$ with $d=0$ and $e \neq 0$. The unipotent automorphisms of height $v=5, t \rightarrow t+Z t^{5+1}$ will not affect this form. As before $v \neq 6,7,8$. By using the device of Lemma 3 we can obtain an equivalent ring of form $C$ with $h=0$. By Lemma 1 we can obtain an equivalent ring with $g=i=0$. Hence any ring in this case is equivalent to a ring of the form

$$
\left(t^{5}, t^{9}+e t^{17}+f t^{21}\right) \text {. }
$$

If there were to be rings of this form equivalent by unipotent automorphisms the height of these would have to be $v>9$. But $t \rightarrow t+Z t^{10+1}$ will not affect this form while unipotent automorphisms of heights 11 and 12 are not allowable because $t^{5}$ would then not be the first generator. Hence none of the above rings can be equivalent by unipotent automorphisms.

Case 3.3. We are here considering rings with generators of form $D$ :

$$
\left(t^{5}, t^{9}+f t^{21}+g t^{22}+h t^{26}+i t^{31}\right) .
$$

Now $f$ is invariant for any unipotent automorphism that leaves this form invariant. We then divide this case into two subcases, Case 3.31: $f \neq 0$ and Case 3.32: $f=0$. 
Case 3.31: form $D$ with $f \neq 0$. By alternate applications of Lemmas 1 and 2 we can obtain an equivalent ring of form $D$ with $g=h=i=0$. Hence the canonical form for Case 3.31 is

$$
\left(t^{5}, t^{9}+f t^{21}\right)
$$

Case 3.32: form $D$ with $f=0$. By Lemma 1 we can take $g=0$. Hence we are considering rings with generators of form $E$ :

$$
\left(t^{5}, t^{9}+h t^{26}+i t^{31}\right) \text {. }
$$

The coefficient $h$ is invariant for any unipotent automorphism that leaves this form invariant. By Lemma 1 we find an equivalent ring of form $E$ with $i=0$. Hence the canonical form for Case 3.32 is

$$
\left(t^{5}, t^{9}+h t^{26}\right) \text {. }
$$

We are now ready to summarize the effect of the group of unipotent automorphisms on the set of rings $\mathfrak{T}$.

SUMmaRY. Any ring in class $\mathfrak{I}$ is equivalent to a ring with generators of one of the following forms:

(1) $\left(t^{5}, t^{9}+a t^{11}+b t^{12}+e t^{17}\right), a \neq 0$.

(2) $\left(t^{5}, t^{9}+b t^{12}+d t^{16}\right), b \neq 0$.

(3) $\left(t^{5}, t^{9}+d t^{16}+e t^{17}\right), d \neq 0$.

(4) $\left(t^{5}, t^{9}+e t^{17}+f t^{21}\right), e \neq 0$.

(5) $\left(t^{5}, t^{9}+f t^{21}\right), f \neq 0$.

(6) $\left(t^{5}, t^{9}+h t^{26}\right), h \neq 0$.

(7) $\left(t^{5}, t^{9}\right)$.

Moreover, none of these can be equivalent by unipotent automorphisms.

Since every automotrphism in $\mathfrak{5}$ is the product of an unipotent automorpism and an automorphism $t \rightarrow \xi t, \xi \neq 0$, it remains to analyze the effect of this latter type of automorphism on the forms listed above.

If the automorphism $t \rightarrow \xi t$ is applied to the ring with generators

$$
\left(t^{5}, t^{9}+a_{1} t^{m_{1}}+\cdots+a_{s} t^{m_{s}}\right),
$$

the image is the ring with generatotrs

$$
\left(t^{5}, t^{9}+\xi^{m_{1}-9} a_{1} t^{m_{1}}+\cdots+\xi^{m_{s}-9} a_{s} t^{m_{s}}\right) .
$$

Canonical forms are found for these automorphisms by the device described in the following lemma.

LEMMA 4. If the affine space $A^{j}$ has coordinates $(x)=\left(x_{1}, x_{2}, \cdots, x_{j}\right)$ and if $U$ is the subset of $A^{j}$ defined by $x_{1} \cdot x_{2} \cdots x_{j} \neq 0$, and if the multiplicative group of the field $\Omega$ operates on $U$ by the formula $\xi(x)=\left(\xi^{v_{1}} x_{1}, \cdots, \xi^{v_{j}} x_{j}\right)$ where each $v_{i}$ is a positive integer, then there exists a polynomial $\phi(x)$ such that each point of $U$ is equivalent to one and only one point of $U \cap(\phi(x)=0)$. 
Proof. First assume $v_{i}=$ g.c.d. $\left(v_{1}, \cdots, v_{j}\right)$. Then take $\phi(x)=x_{i}-1$. $\phi(\xi(x))=\xi^{v_{i}} x_{i}-1$ and for $(a)$ in $U$, if $\xi=\left(1 / a_{i}\right)^{1 / v_{i}}, \phi(\xi(a))=0$. Moreover, $\phi(a)=\phi(\xi(a))=0$ implies $\xi^{v_{k}}=1$ for each $v_{k}$ and hence $\xi(a)=(a)$.

Now assume $k=$ g.c.d. $\left(v_{1}, \cdots, v_{j}\right)$ and $k$ is not one of the $v_{i}$ 's. Write $k$ in the form

$$
k=\mu_{i_{1}} v_{i_{1}}+\cdots+\mu_{i_{r}} v_{i_{r}}-\mu_{j_{1}} v_{j_{1}}-\cdots-\mu_{j_{s}} v_{j_{s}}
$$

where the $\mu$ 's are positive integers. Now let

$$
\phi(x)=x_{i_{1}}^{\mu_{i_{1}}} \cdots x_{i_{r}}^{\mu_{i r}}-x_{j_{1}}^{\mu_{i_{1}}} \cdots x_{j_{s}}^{\mu_{j_{s}}} .
$$

Then we have for any $(a)$ in $U$ and any $\xi \neq 0$ in $\Omega$,

$$
\phi(\xi(a))=\xi^{l}\left[\xi^{k} a_{i_{1}}^{\mu_{i_{1}}} \cdots a_{i_{r}}^{\mu_{i r}}-a_{j_{1}}^{\mu_{j_{1}}} \cdots a_{j_{s}}^{\mu_{j_{\ell}}}\right] .
$$

Clearly $\xi$ can be chosen so that $\phi(\xi(a))=0$. Moreover $\phi(b)=0$ and $\phi(\xi(b))=0$ implies $\xi^{k}=1$. Then $\xi(b)=(b)$. Q.E.D.

Applying the procedure of Lemma 4 to the forms computed previously, we have the following conclusion:

THEOREM. Any ring in class $\mathfrak{I}$ is equivalvnt to one and only one ring with generators of one of the following canonical forms:

(1) $\left(t^{5}, t^{9}+a t^{11}+a t^{12}+e t^{17}\right), a \neq 0$ and e any constant, $\left(t^{5}, t^{9}+t^{11}+e t^{17}\right), e$ any constant.

(2) $\left(t^{5}, t^{9}+b t^{12}+b^{2} t^{16}\right), b \neq 0$; $\left(t^{5}, t^{9}+t^{12}\right)$

(3) $\left(t^{5}, t^{9}+d t^{16}+d t^{17}\right), d \neq 0$; $\left(t^{5}, t^{9}+t^{16}\right)$.

(4) $\left(t^{5}, t^{9}+e t^{17}+e t^{21}\right), e \neq 0$; $\left(t^{5}, t^{9}+t^{17}\right)$

(5) $\left(t^{5}, t^{9}+t^{21}\right)$.

(6) $\left(t^{5}, t^{9}+t^{26}\right)$.

(7) $\left(t^{5}, t^{9}\right)$.

4. A listing of canonical forms for the simplest cases. This section will include a listing of canonical forms for the complete local subrings $\mathfrak{D}$ of $\Omega[[t]]$ for certain special cases. The characteristic is assumed to be 0 . The computational methods by which these representative rings were obtained are fully illustrated in the previous section.

First we must introduce some notation. If the smallest integer in the semigroup $G(\mathfrak{D})$ is $n$, we will say that $\mathfrak{D}$ is of order $n$ and the corresponding singular point may be termed a cusp of order $n$. The numerical invariant $\operatorname{dim}_{\Omega} M / M^{2}$ equals the number of elements in a minimal set of generators for $\mathfrak{D}$. If $\operatorname{dim}_{\Omega} M / M^{2}=2$, there will be a plane curve having $\mathfrak{D}$ as the complete local ring of one of its singular points. 
Let $m$ denote the smallest integer in $G(\mathfrak{D})$ that is not a multiple of $n$ and in case $\operatorname{dim}_{\Omega} M / M^{2}=3$, let $r$ be the third integer in $G(\mathfrak{D})$ such that there are no elements in $M^{2}$ of order $r$.

We now list representative rings for all cases for which $n=2$ or 3 and also for $n=4, m<12$ in the case of plane curves. Of course we list the rings by listing generators.

$$
\begin{aligned}
& n=2, m \text { an odd integer: }\left(t^{2}, t^{m}\right) \text {. } \\
& n=3: \operatorname{dim}_{\Omega} M / M^{2}=2 \text {. } \\
& m=4:\left(t^{3}, t^{4}\right) \text {. } \\
& m=5:\left(t^{3}, t^{5}\right) \text {. } \\
& m=7:\left(t^{3}, t^{7}\right),\left(t^{3}, t^{7}+t^{8}\right) \text {. } \\
& m=8:\left(t^{3}, t^{8}\right),\left(t^{3}, t^{8}+t^{10}\right) \text {. } \\
& m=3 q+1, q>2:\left(t^{3}, t^{3 q+1}\right),\left(t^{3}, t^{3 q+1}+t^{3 q+2}\right),\left(t^{3}, t^{3 q+1}+t^{3(q+1)+2}\right), \cdots, \\
& \left(t^{3}, t^{3 q+1}+t^{3(2 q-2)+2}\right) \text {. } \\
& m=3 q+2, q>2:\left(t^{3}, t^{3 q+2}\right),\left(t^{3}, t^{3 q+2}+t^{3(q+1)+1}\right),\left(t^{3}, t^{3 q+2}+t^{3(q+2)+1}\right), \cdots, \\
& \left(t^{3}, t^{3 q+2}+t^{3(2 q-1)+1}\right) \text {. } \\
& n=3: \operatorname{dim}_{\Omega} M / M^{2}=3 \text {. } \\
& m=4, r=5:\left(t^{3}, t^{4}, t^{5}\right) \text {. } \\
& m=5, r=7:\left(t^{3}, t^{5}, t^{7}\right) \text {. } \\
& m=7, r=8:\left(t^{3}, t^{7}, t^{8}\right) \text {. } \\
& m=7, r=11:\left(t^{3}, t^{7}, t^{11}\right),\left(t^{3}, t^{7}+t^{8}, t^{11}\right) \text {. } \\
& m=8, r=10:\left(t^{3}, t^{8}, t^{10}\right) \text {. } \\
& m=8, r=13:\left(t^{3}, t^{8}, t^{13}\right),\left(t^{3}, t^{8}+t^{10}, t^{13}\right) \text {. } \\
& m=3 q+1, q>2:\left(t^{3}, t^{3 q+1}, t^{3(q+i)+2}\right),\left(t^{3}, t^{3 q+1}+t^{3(q+1)+2}, t^{3(q+i)+2}\right), \cdots, \\
& \left(t^{3}, t^{3 q+1}+t^{3(q+i-1)+2}, t^{3(q+i)+2}\right) \text {. } \\
& r=3(q+i)+2 \text {. } \\
& 0 \leqq i \leqq q-1 \text {. } \\
& m=3 q+2, q>2:\left(t^{3}, t^{3 q+2}, t^{3(q+i)+1}\right),\left(t^{3}, t^{3 q+2}+t^{3(q+1)+1}, t^{3(q+i)+1}\right), \cdots, \\
& r=3(q+i)+1 \text {. } \\
& \left(t^{3}, t^{3 q+2}+t^{3(q+i-1)+1}, t^{3(q+i)+1}\right) \text {. } \\
& 1 \leqq i \leqq q \text {. }
\end{aligned}
$$

We will now list generators for the representative rings for $n=4$ and $\operatorname{dim}_{\Omega} M / M^{2}=2$.

$$
\begin{aligned}
& m=5:\left(t^{4}, t^{5}\right),\left(t^{4}, t^{5}+t^{7}\right) \\
& m=6:\left(t^{4}, t^{6}+t^{v}\right) \text { where } v \text { is any odd integer }>6 . v \text { is a numerical invariant } \\
& \quad \text { and the degree of the conductor } d \text { is a function of } v . \\
& m=7:\left(t^{4}, t^{7}\right),\left(t^{4}, t^{7}+t^{9}\right),\left(t^{4}, t^{7}+t^{13}\right) \\
& m=9:\left(t^{4}, t^{9}+t^{10}+a t^{11}\right), a \text { any constant } \neq 18 / 19 \\
& \left(t^{4}, t^{9}+t^{10}+\frac{18}{19} t^{11}+b t^{15}\right), b \text { any constant; } \\
& \left(t^{4}, t^{9}+t^{15}\right),\left(t^{4}, t^{9}+t^{19}\right),\left(t^{4}, t^{9}\right) .
\end{aligned}
$$


$m=10$ : Any such ring $\mathfrak{D}$ is equivalent to a ring with generators of the form $\left(t^{4}, t^{10}+c t^{v}+\cdots\right)$ where $v$ is some odd integer. $v$ is a numerical invariant. Hence we consider each case separately.

$$
\begin{aligned}
& m=10, v=11:\left(t^{4}, t^{10}+t^{11}+b t^{13}\right) \text { where } b \text { is any constant. } \\
& v=13:\left(t^{4}, t^{10}+a t^{13}+b t^{15}\right) \text { where } a \neq 0, a^{2}-b=0 \text {, and } \\
& \left(t^{4}, t^{10}+t^{13}\right) \text {. } \\
& v=10+2 j+1:\left(t^{4}, t^{10}+a t^{10+2 j+1}+b t^{10+2 j+3}\right) \quad \text { where } a \neq 0 \text {, } \\
& a^{j+1}-b^{j}=0, j=1,2, \cdots \text { and }\left(t^{4}, t^{10}+t^{10+2 j+1}\right) . \\
& m=11:\left(t^{4}, t^{11}+a t^{13}+a t^{14}\right), a \text { any nonzero constant. } \\
& \left(t^{4}, t^{11}+a t^{14}+a^{2} t^{18}\right), a \neq 0 . \\
& \left(t^{4}, t^{11}+t^{14}\right),\left(t^{4}, t^{11}+t^{17}\right),\left(t^{4}, t^{11}+t^{21}\right),\left(t^{4}, t^{11}+t^{25}\right),\left(t^{4}, t^{11}\right) .
\end{aligned}
$$

Canonical forms for these local rings had received study previously in a paper by Kasner and DeCicco [4]. They studied only the case of plane curves (i.e. $\left.\operatorname{dim}_{\Omega} M / M^{2}=2\right)$ and called the pair of integers $(n, m)$ a species. Their main result in [4] was to determine which species had only finitely many representations and which species contained at least a one-parameter family of rings, none of which were equivalent.

For $n=2$ and 3 there are only finitely many forms corresponding to each species. The species $(4,5)$ and $(4,7)$ are also finite. The species $(4,6)$ may be subdivided into subspecies by the invariant $v$ mentioned above. Each subspecies has only one representative. Then it can be shown that for all other species, the canonical forms contain at least one one-parameter family of rings, no two of which are equivalent by the group $(5$.

Another invariant which is useful in measuring the complexity of cusps is $\delta=\operatorname{dim}_{\Omega} \overline{\mathfrak{D}} / \mathfrak{D}$, where $\overline{\mathfrak{D}}$ is the integral closure of $\mathfrak{D}$. This is called the degree of the singularity. We will now list all complete local rings for cusps for which $\delta \leqq 6$. We note that the set of representative rings for $\delta<6$ is finite while $\delta=6$ contains a one-parameter family of rings.

$$
\begin{aligned}
\delta=1: & \left(t^{2}, t^{3}\right) . \\
\delta=2: & \left(t^{2}, t^{5}\right),\left(t^{3}, t^{4}, t^{5}\right) . \\
\delta=3: \quad & \left(t^{2}, t^{7}\right),\left(t^{3}, t^{4}\right),\left(t^{3}, t^{5}, t^{7}\right),\left(t^{4}, t^{5}, t^{6}, t^{7}\right) . \\
\delta=4: \quad & \left(t^{2}, t^{9}\right),\left(t^{3}, t^{5}\right),\left(t^{3}, t^{7}, t^{8}\right),\left(t^{4}, t^{5}, t^{6}\right),\left(t^{4}, t^{5}, t^{7}\right),\left(t^{4}, t^{6}, t^{7}, t^{9}\right), \\
& \left(t^{5}, t^{6}, t^{7}, t^{8}, t^{9}\right) . \\
\delta=5: \quad & \left(t^{2}, t^{11}\right),\left(t^{3}, t^{7}, t^{11}\right),\left(t^{3}, t^{7}+t^{8}, t^{11}\right),\left(t^{3}, t^{8}, t^{10}\right),\left(t^{4}, t^{5}, t^{11}\right), \\
& \left(t^{4}, t^{5}+t^{7}, t^{11}\right),\left(t^{4}, t^{6}, t^{7}\right),\left(t^{4}, t^{6}, t^{9}, t^{11}\right),\left(t^{4}, t^{6}+t^{7}, t^{9}, t^{11}\right), \\
& \left(t^{4}, t^{7}, t^{9}, t^{10}\right),\left(t^{5}, t^{6}, t^{7}, t^{8}\right),\left(t^{5}, t^{6}, t^{7}, t^{9}\right),\left(t^{5}, t^{6}, t^{8}, t^{9}\right), \\
& \left(t^{5}, t^{7}, t^{8}, t^{9}, t^{11}\right),\left(t^{6}, t^{7}, t^{8}, t^{9}, t^{10}, t^{11}\right) .
\end{aligned}
$$




$$
\begin{aligned}
\delta=6: & \left(t^{2}, t^{13}\right),\left(t^{3}, t^{7}\right),\left(t^{3}, t^{7}+t^{8}\right),\left(t^{3}, t^{8}, t^{13}\right),\left(t^{3}, t^{8}+t^{10}, t^{13}\right),\left(t^{4}, t^{5}\right), \\
& \left(t^{4}, t^{5}+t^{7}\right),\left(t^{4}, t^{6}, t^{9}\right),\left(t^{4}, t^{6}+t^{7}, t^{11}\right),\left(t^{4}, t^{6}, t^{11}, t^{13}\right), \\
& \left(t^{4}, t^{6}+t^{9}, t^{11}, t^{13}\right),\left(t^{4}, t^{7}, t^{9}\right),\left(t^{4}, t^{7}, t^{9}+t^{10}\right),\left(t^{4}, t^{9}, t^{10}, t^{11}\right), \\
& \left(t^{5}, t^{6}, t^{7}\right),\left(t^{5}, t^{6}+t^{9}, t^{7}\right),\left(t^{5}, t^{6}+t^{8}, t^{7}\right),\left(t^{5}, t^{6}+a t^{8}+a t^{9}, t^{7}\right), \\
& \text { where } a \text { is any nonzero constant, }\left(t^{5}, t^{6}, t^{8}\right),\left(t^{5}, t^{6}+t^{7}, t^{8}\right), \\
& \left(t^{5}, t^{6}, t^{9}, t^{13}\right),\left(t^{5}, t^{7}, t^{8}, t^{9}\right),\left(t^{5}, t^{7}, t^{8}, t^{11}\right),\left(t^{5}, t^{7}, t^{8}+t^{9}, t^{11}\right), \\
& \left(t^{5}, t^{7}, t^{9}, t^{11}, t^{13}\right),\left(t^{5}, t^{7}+t^{8}, t^{9}, t^{11}, t^{13}\right),\left(t^{5}, t^{8}, t^{9}, t^{11}, t^{12}\right), \\
& \left(t^{6}, t^{7}, t^{8}, t^{9}, t^{10}\right),\left(t^{6}, t^{7}, t^{8}, t^{9}, t^{11}\right),\left(t^{6}, t^{7}, t^{8}, t^{10}, t^{11}\right), \\
& \left(t^{6}, t^{7}, t^{9}, t^{10}, t^{11}\right),\left(t^{6}, t^{8}, t^{9}, t^{10}, t^{11}, t^{13}\right),\left(t^{7}, t^{8}, t^{9}, t^{10}, t^{11}, t^{12}, t^{13}\right) .
\end{aligned}
$$

5. Remarks concerning field characteristic and multiple-branch singularities. In this section we will briefly discuss the significance of the field characteristic.

For most characteristics $p \neq 0$ the forms will remain the same as for $p=0$. However it is not just in the case of $p$ dividing $n$ or $m$ that the forms will change. For example the species $(4,9)$ contains the canonical form

$$
\left(t^{4}, t^{9}+t^{10}+\frac{18}{19} t^{11}+b t^{15}\right)
$$

Clearly this is changed for $p=19$.

The most striking changes occur when $p$ does divide $n$ or $m$. If $p \mid n$, then the first generator cannot always be taken in the form $t^{n}$. Consider the species $(3,8)$. For the usual case there are only two nonequivalent forms: $\left(t^{3}, t^{8}\right)$ and $\left(t^{3}, t^{8}+t^{10}\right)$. For characteristic $p=3$, the canonical forms are:

$$
\begin{aligned}
& \left(t^{3}+t^{4}, t^{8}\right),\left(t^{3}+t^{5}+c t^{7}, t^{8}\right) c \text { arbitrary, }\left(t^{3}+t^{7}, t^{8}\right), \\
& \left(t^{3}+b t^{5}+b^{2} t^{7}+b^{3} t^{10}, t^{8}\right) b \text { arbitrary except } b \neq 0,\left(t^{3}+t^{10}, t^{8}\right) \\
& \left(t^{3}+t^{13}, t^{8}\right),\left(t^{3}, t^{8}\right) .
\end{aligned}
$$

Notice that we have here two one-parameter families among the representatives.

The purpose of this final section is to discuss the case of "multiple-branch" singularities. First we recall what is meant by this terminology. If $C$ is a curve, $P$ a singular point of $C$, and $\mathfrak{D}_{P}$ the local ring of $C$ at $P$, the number of branches of $C$ at $P$ means the number of nontrivial valuation rings of $\Omega(C)$ which contain $\mathfrak{b}_{P}$.

We will now describe the complete local ring for a two-branch singularity at $P$. Let $R_{1}$ and $R_{2}$ be the two valuation rings of $\Omega(C)$ containing $\mathfrak{D}_{P}$. The integral closure of $\mathrm{D}_{P}$ is $S=R_{1} \cap R_{2}$. Since $\mathfrak{D}_{P}$ is a subspace of $S, \mathfrak{o}_{P}^{*}$ the completion of $\mathfrak{D}_{P}$ will be a subring and subspace of $S^{*}$, the completion of $S$.

It is shown in [2] that $S^{*}$ is the direct product of the completions of $R_{1}$ and $R_{2}$. Hence $S^{*}$ is isomorphic to the direct product $\Omega[[s]] \times \Omega[[t]]$ where $s$ and $t$ are indeterminates. There exists a conductor, i.e. integers $d$ and $d^{\prime}$ such that $\left(s^{v}, 0\right)$ and $\left(0, t^{\mu}\right)$ are in $\mathfrak{o}_{P}^{*}$ for $v \geqq d, \mu \geqq d^{\prime}$. Moreover a pair of power series $(\phi(s), \theta(t))$ in $\mathfrak{o}_{P}^{*}$ must satisfy $\phi(0)=\theta(0)$.

Thus we may consider $\mathfrak{D}_{P}^{*}$ as a subring of the power series ring $\Omega[[s, t]]$ where 
we define the product $s \cdot t=0$. There is an integer $N$ so that all powers of $s$ and $t \geqq N$ are in $\mathfrak{D}_{P}^{*}$.

Let $\mathfrak{L}$ be the group of $\Omega$-automorphisms of $\Omega[[s, t]]$ where $s \cdot t=0$. The automorphisms of $\mathfrak{L}$ are of two types:

$$
\begin{array}{ll}
s \rightarrow s^{\prime}=\phi(s), & t \rightarrow t^{\prime}=\theta(t), \\
s \rightarrow s^{\prime}=\phi(t), & t \rightarrow t^{\prime}=\theta(s)
\end{array}
$$

where $\phi$ and $\theta$ are power series of order one. Consider the class $\mathfrak{I}$ of all those rings containing all powers of $s$ and $t$ that are $\geqq N$. Define $\mathfrak{L}_{N}$ as the subgroup of automorphisms of the form

$$
s \rightarrow s+a s^{N}+\cdots, \quad t \rightarrow t+b t^{N}+\cdots .
$$

Then $\mathfrak{L} / \mathfrak{L}_{N}$ is a solvable algebraic group whose operation on the rings of class $\mathfrak{T}$ coincides with the operation of $\mathfrak{L}$. However $\mathfrak{L} / \mathfrak{L}_{N}$ is not connected. The two connected components of $\mathfrak{L} / \mathfrak{L}_{N}$ correspond to the two types of automorphisms mentioned above.

Since the machinery we used from the theory of algebraic groups depended on the groups being solvable and connected, the approach we used in $\S \S 1$ and 2 cannot be carried over to multiple-branch singularities.

\section{REFERENCES}

1. A. Borel, Groupes linéaires algébriques, Ann. of Math. (2) 64 (1956), 20-82.

2. C. Chevalley, On the theory of local rings, Ann. of Math. (2) 43 (1943), 690-708.

3. - Sur la théorie des variétés algébriques, Nagoya Math. J. 8 (1955), 1-44.

4. E. Kasner and J. DeCicco, The general invariant theory of irregular analytic arcs or elements, Trans. Amer. Math. Soc. 51 (1942), 232-254.

5. M. Rosenlicht, Some basic theorems on algebraic groups, Amer. J. Math. 78 (1956), 401-443.

6. - Equivalence relations on algebraic curves, Ann. of Math. (2) 56 (1952), 169-191.

7. A. Weil, On algebraic groups of transformations, Amer. J. Math. 77 (1955), 355-391.

MERCER UNIVERSITY, Macon, Georgia 